\title{
Bessel-Beam Photoacoustic Microscopical Simulation Platform Based on k-Wave
}

\author{
Xianlin Song ${ }^{* \#}$, Jianshuang Wei ${ }^{2 \#}$ \\ ${ }^{1}$ School of Information Engineering, Nanchang University, Nanchang, China \\ ${ }^{2}$ Britton Chance Center for Biomedical Photonics, Wuhan National Laboratory for Optoelectronics, Huazhong University of \\ Science and Technology, Wuhan, China \\ Email: *songxianlin@ncu.edu.cn
}

How to cite this paper: Song, X.L. and Wei, J.S. (2020) Bessel-Beam Photoacoustic Microscopical Simulation Platform Based on k-Wave. Optics and Photonics Journal, 10, 111-116.

https://doi.org/10.4236/opj.2020.106011

Received: March 25, 2020

Accepted: June 26, 2020

Published: June 29, 2020

\begin{abstract}
We developed a Bessel-beam photoacoustic microscopical simulation platform by using the k-Wave: MATLAB toolbox. The simulation platform uses the ring slit method to generate Bessel beam. By controlling the inner and outer radius of the ring slit, the depth-of-field $(\mathrm{DoF})$ of Bessel beam can be controlled. And the large volumetric image is obtained by point scanning. The simulation experiments on blood vessels were carried out to demonstrate the feasibility of the simulation platform. This simulation work can be used as an auxiliary tool for the research of Bessel-beam photoacoustic microscopy.
\end{abstract}

\section{Keywords}

k-Wave, Bessel Beam, Photoacoustic Microscope

\section{Introduction}

Photoacoustic imaging is a promising technique that combines optical contrast with ultrasonic detection to map the distribution of the absorbing pigments in biological tissues [1] [2] [3] [4]. It has been widely used in biological researches, such as structural imaging of vasculature [5], brain structural and functional imaging [6], and tumor detection [7]. Considering the lateral resolution of photoacoustic microscopy (PAM), it can be classified into two categories: optical-resolution (OR-) and acoustic-resolution (AR-) PAM [8] [9]. In AR-PAM, the spatial resolution is determined by the acoustic focus, since the laser light is weekly or even not focused on the sample. Conversely, in the OR-PAM, the laser light is tightly focused into the sample to achieve sharp excitation. However, in

${ }^{\#}$ Equally contributed to this work. 
OR-PAM, the DoF is quite limited, because it is determined by the optical condenser and is closely related to the optical focusing. The small DoF will prevent OR-PAM to achieve high-quality 3D images or acquire dynamic information in depth direction.

Depth scanning using motorized stage is widely used to address this issue as it is the most convenient method [10] [11]. To avoid slow mechanical scanning in the depth direction, several methods by engineering the illumination have been proposed. Double illumination can double the DoF by illuminating the sample from both top and bottom sides simultaneously. But it is only valid in transmission-mode OR-PAM [12]. Utilizing chromatic aberration of non-achromatic objective, multi-wavelength laser can generate multi-focus along the depth direction [13]. However, this method sacrifices the capability of functional imaging. Electrically tunable lens (ETL) has also been introduced in OR-PAM [14]. The focal plane can be settled in about $15 \mathrm{~ms}$ (EL-10-30, Optotune AG). It is fast enough for pulsed lasers with a repetition rate of hundred-hertz, while being quite slow for those lasers with repetition rate of hundreds of kilo-hertz.

Non-diffraction beam inherently owns a large DoF. Photoacoustic Microscopy Based on Bessel Beam can achieve extended DoF with retaining high lateral resolution [15]. In this paper, we developed a Bessel beam photoacoustic microscopical simulation platform with extended DoF by using the k-Wave: MATLAB toolbox. The simulation platform uses the ring slit method to generate Bessel beam. By controlling the inner and outer radius of the ring slit, the DoF of Bessel beam can be controlled. And the large volumetric image is obtained by point scanning. And the simulation experiments on blood vessels were carried out to demonstrate the feasibility of simulation platform.

\section{Simulation Method}

\subsection{Configuration Environment}

The k-Wave simulation toolbox can analyze photoacoustic signals in the time domain [16]. We use k-Wave: MATLAB toolbox for the simulation of Bessel-beam photoacoustic microscopy. The simulated environment was created in three dimension with $100 \times 100 \times 100$ voxels (each voxel size is $2 \mu \mathrm{m}$ ), as shown in Figure 1, and contains a perfectly matched boundary layer (PML) to satisfy the boundary conditions for the forward process. The surrounding medium is water with a sound velocity of $1.5 \mathrm{~km} / \mathrm{s}$ and a density of $1000 \mathrm{~kg} / \mathrm{m}^{3}$. All simulations assume that an acoustically homogeneous medium was considered with no absorption or dispersion of sound.

In our simulation, the parameters of the simulated system were chosen to match those of the practical setup as closely as possible, the center frequency of the ultrasonic transducer is set to $50 \mathrm{MHz}$ and the bandwidth is $80 \%$. The three-dimensional imaging data can be obtained by carried out two-dimensional raster scan. 


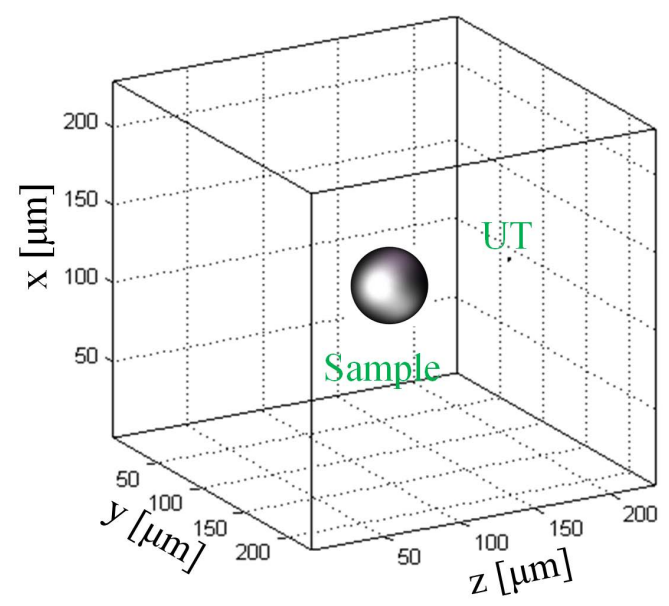

Figure 1. Schematic diagram of simulation of Bessel-beam photoacoustic microscopy.

\subsection{Generation of Bessel Beams}

Based on Fourier optics theory, since the Fourier transform of a ring is a zero-order Bessel function, placing a ring slit on the back focal plane of the lens can convert incident light into a Bessel beam. If the annular slit is symmetrical about the optical axis, the field intensity distribution near the front focal plane can be known from the scalar diffraction theory

$$
E(r, z)=A \frac{e^{i \kappa z}}{i \lambda f} \int P(\rho) J_{0}\left(\frac{\kappa}{f} r \rho\right) e^{-i \frac{\rho^{2}}{f^{2}} \kappa z} \rho d \rho
$$

where $P(\rho)$ is the pupil function, $f$ is the lens focal length, $\lambda$ is the wavelength, and $A$ is a constant representing the amplitude. $P(\rho)$ is a circular slit and substituting it into the above formula, the distribution of the approximate Bessel function can be obtained. The field distribution of the slit with an outer ring radius of $5 \mathrm{~mm}$ near the focal point of the objective lens with a focal length of $40 \mathrm{~mm}$ is shown in Figure 2. Figure 2(a) is the Bessel beam generating from an annular slit with an outer ring radius of $5 \mathrm{~mm}$ and a slit width of $150 \mu \mathrm{m}$. Figure 2(b) is the Bessel beam generating from an annular slit with an outer ring radius of $5 \mathrm{~mm}$ and a slit width of $400 \mu \mathrm{m}$. As the slit width increases, the DoF decreases. Figure 2 (c) is the intensity distribution in focal plane indicated by the yellow dashed line in Figure 2(b). The lateral resolution can be calculated from the full height at half maximum (FWHM) of the intensity distribution of Bessel beam in focal plane, and the FWHM is $\sim 1.2 \mu \mathrm{m}$.

\section{Simulation Results-Imaging of the Vasculature}

A virtual vascular network is used to verify the imaging capabilities of the constructed simulation platform, as shown in Figure 3(a). The diameter of the blood vessel is $2-10 \mu \mathrm{m}$. The blood vessel was placed at a depth of about $300 \mu \mathrm{m}$ from the ultrasonic transducer, and a two-dimensional raster scan was performed with a step size of $2 \mu \mathrm{m}$ to obtain three-dimensional imaging data. Figure 3(b) is 


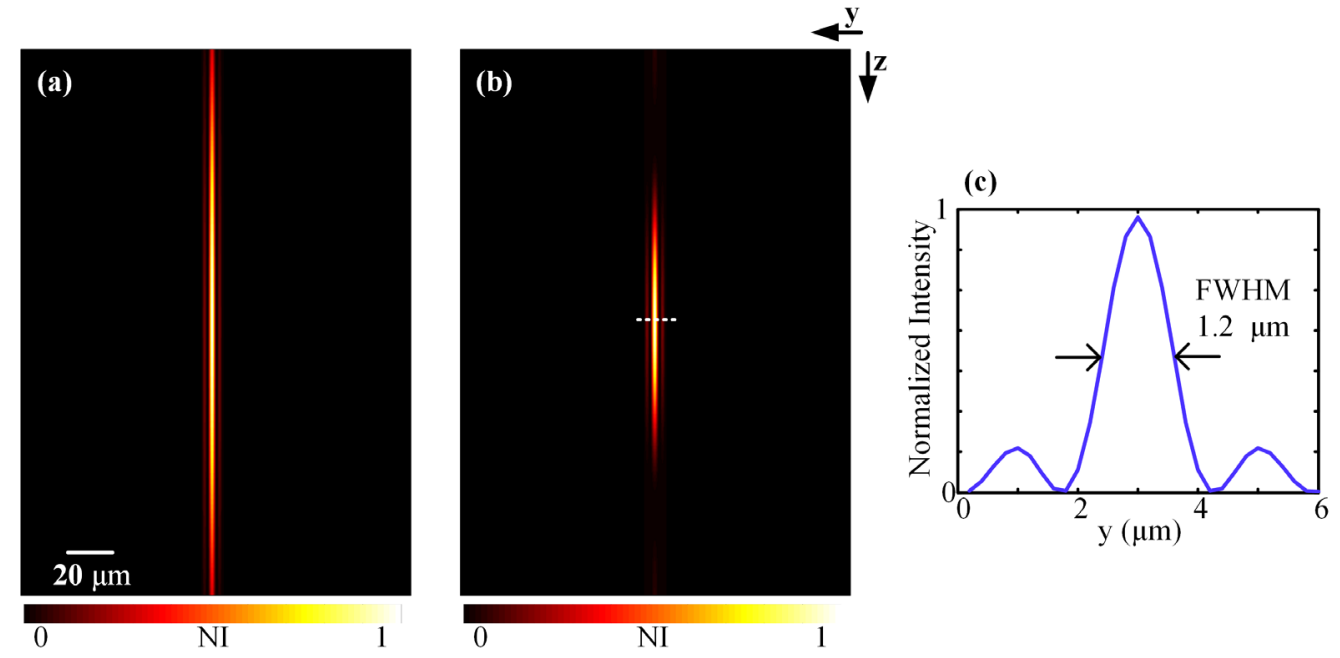

Figure 2. Bessel beam generating from an annular slit with different slit width. (a) Bessel beam generating from an annular slit with an outer ring radius of $5 \mathrm{~mm}$ and a slit width of $150 \mu \mathrm{m}$. (b) Bessel beam generating from an annular slit with an outer ring radius of $5 \mathrm{~mm}$ and a slit width of $400 \mu \mathrm{m}$. (c) Intensity distribution in focal plane indicated by the yellow dashed line in (b). Objective focal length 40 mm, wavelength $582 \mathrm{~nm}$. NI, normalized intensity.
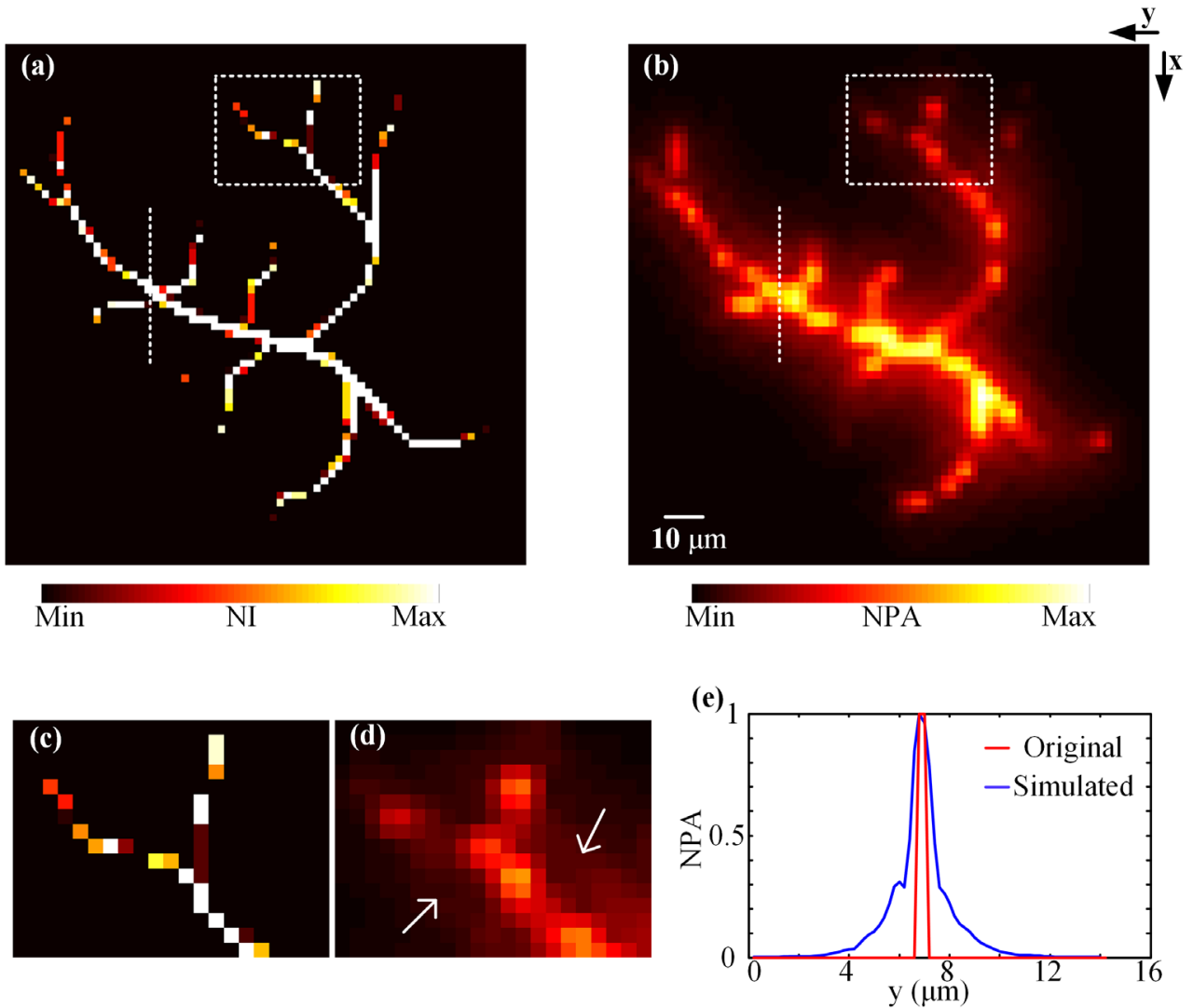

Figure 3. Images of vasculature. (a) Original blood vessels for simulation. (b) The MAP images of vasculature. (c) and (d) are the close-up images of the small areas indicated by the white dashed rectangles in (a) and (b), respectively. (e) is the photoacoustic signal distribution of a vessel (indicated by the white dashed line in (a) and (b)). NPA, normalized photoacoustic amplitude. 
MAP image. Figure 3(c) and Figure 3(d) are the close-up images of the small areas indicated by the white dashed rectangles in Figure 3(a) and Figure 3(b), respectively. The structure of blood vessels can be distinguished, but the image appears blurred due to the side lobe of Bessel beams (indicated by the white arrows in Figure 3(d)). To quantitatively demonstrate the performance of our simulation platform, the photoacoustic signal distribution of a vessel (indicated by the white dashed line in Figure 3(a) and Figure 3(b)) was chosen for width analysis, the corresponding width of the vessel was defined as the FWHM of the photoacoustic signal curve. As shown in Figure 3(e), the width of original vessel is $\sim 2 \mu \mathrm{m}$, while, since the side lobe of Bessel beams can deteriorate the resolution, the width of vessel is measured to be $\sim 9.5 \mu \mathrm{m}$.

\section{Conclusion}

We have developed a Bessel-beam photoacoustic microscopical simulation platform using k-Wave simulation toolbox. Bessel beam was generated from annular slit. And the changes in slit width cause changes in DoF, larger slit width generated narrow DoF. The slit width can be set as experimental requirement. Two-dimensional raster scan was performed to obtained volumetric data. A virtual vascular network is used to verify the imaging capabilities of the constructed simulation platform. This work will contribute to the study of Bessel-beam photoacoustic microscopy.

\section{Conflicts of Interest}

The authors declare no conflicts of interest regarding the publication of this paper.

\section{References}

[1] Wang, L.V. and Hu, S. (2012) Photoacoustic Tomography: In Vivo Imaging from Organelles to Organs. Science, 335, 1458-1462. https://doi.org/10.1126/science.1216210

[2] Wang, L.V., Wang, X., Ku, G. and Stoica, G. (2004) High-Resolution Functional Photoacoustic Tomography. IEEE International Symposium on Biomedical Imaging from Nano to Macro, 1, 1479-1481.

[3] Yao, J. and Wang, L.V. (2011) Photoacoustic Tomography: Fundamentals, Advances and Prospects. Contrast Media Mol. Imaging, 6, 332-345. https://doi.org/10.1002/cmmi.443

[4] Wang, L.V. (2008) Prospects of Photoacoustic Tomography. Medical Physics, 35, 5758-5767. https://doi.org/10.1118/1.3013698

[5] Hu, S. and Wang, L.V. (2010) Photoacoustic Imaging and Characterization of the Microvasculature. Journal of Biomedical Optics, 15, 011101. https://doi.org/10.1117/1.3281673

[6] Wang, X., Pang, Y., Ku, G., Xie, X., Stoica, G. and Wang, L.V. (2003) Noninvasive Laser-Induced Photoacoustic Tomography for Structural and Functional in Vivo Imaging of the Brain. Nature Biotechnology, 21, 803-806.

https://doi.org/10.1038/nbt839 
[7] Zhang, H.F., Maslov, K., Stoica, G. and Wang, L.V. (2006) Functional Photoacoustic Microscopy for High-Resolution and Noninvasive in Vivo Imaging. Nature Biotechnology, 24, 848-851. https://doi.org/10.1038/nbt1220

[8] Liu, Y., Zhang, C. and Wang, L.V. (2012) Effects of Light Scattering on Optical-Resolution Photoacoustic Microscopy. Journal of Biomedical Optics, 17, Article ID: 126014. https://doi.org/10.1117/1.JBO.17.12.126014

[9] Maslov, K., Zhang, H.F., Hu, S. and Wang, L.V. (2008) Optical-Resolution Photoacoustic Microscopy for in Vivo Imaging of Single Capillaries. Optics Letters, 33, 929-931. https://doi.org/10.1364/OL.33.000929

[10] Yao, J., Wang, L., Yang, J.M., Maslov, K.I., Wong, T.T.W., Li, L., Huang, C.H., Zou, J. and Wang, L.V. (2015) High-Speed Label-Free Functional Photoacoustic Microscopy of Mouse Brain in Action. Nature Methods, 12, 407-410. https://doi.org/10.1038/nmeth.3336

[11] Yeh, C., Soetikno, B., Hu, S., Maslov, K.I. and Wang, L.V. (2014) Microvascular Quantification Based on Contour-Scanning Photoacoustic Microscopy. Journal of Biomedical Optics, 19, Article ID: 096011. https://doi.org/10.1117/1.JBO.19.9.096011

[12] Yao, J., Maslov, K.I., Puckett, E.R., Rowland, K.J., Warner, B.W. and Wang, L.V. (2012) Double-Illumination Photoacoustic Microscopy. Optics Letters, 37, 659-661. https://doi.org/10.1364/OL.37.000659

[13] Hajireza, P., Forbrich, A. and Zemp, R.J. (2013) Multifocus Optical-Resolution Photoacoustic Microscopy Using Stimulated Raman Scattering and Chromatic Aberration. Optics Letters, 38, 2711-2713. https://doi.org/10.1364/OL.38.002711

[14] Li, B., Qin, H., Yang, S. and Xing, D. (2014) In Vivo Fast Variable Focus Photoacoustic Microscopy Using an Electrically Tunable Lens. Opt. Express., 22, Article ID: 020130. https://doi.org/10.1364/OE.22.020130

[15] Shi, J., Wang, L., Noordam, C. and Wang, L.V. (2015) Bessel-Beam Grueneisen Relaxation Photoacoustic Microscopy with Extended Depth of Field. Journal of Biomedical Optics, 20, Article ID: 116002. https://doi.org/10.1117/1.JBO.20.11.116002

[16] Treeby, B.E. and Cox, B.T. (2010) k-Wave: MATLAB Toolbox for the Simulation and Reconstruction of Photoacoustic Wave Fields. Journal of Biomedical Optics, 15, 021314. https://doi.org/10.1117/1.3360308 